\title{
A Correction Scheme For Point-Particle Modeling of Droplets and Spray Systems
}

\author{
Sourabh V. Apte*1 \\ ${ }^{1}$ Mechanical Engineering, Oregon State University, Corvallis, OR, USA \\ *Corresponding author email: sva@oregonstate.edu
}

\begin{abstract}
Modeling of spray systems using the point-particle approach requires estimation of the undisturbed fluid flow quantities such as velocity, pressure, species mass fraction, and temperature at the droplet location, to accurately capture the droplet dynamics. However, in a typical two-way coupled computation, the droplets affect the fluid flow through mass, momentum, and energy exchange, and disturb the flow. This self-disturbance effect is significant for droplet sizes that are on the same order or larger than the grid resolution, which in practice is common in spray studies, even for a point-particle based approach. A general formulation that accounts for the self-disturbance created by the dispersed droplets in obtaining the undisturbed fluid flow for accurate computation of the force closure, thermal heating, and evaporation is derived. This self disturbance-corrected approach is evaluated for two simple test cases of (i) single droplet held fixed in a uniform flow of hot fluid, and (ii) gravitational settling of a droplet in a quiescent, hot fluid to show very good predictive capability. The approach is straightforward and can be applied to any numerical formulation for spray systems.
\end{abstract}

\section{Keywords}

Self-disturbance correction, point-particle, undisturbed flow.

\section{Introduction}

Modeling of spray systems typically employs the the point-particle (PP) approach [1], in which the spray droplets are assumed spherical, subgrid, with low volume loading, and modeled as point sources. These assumptions also facilitate use of standard closure models for drag force, heat and mass transfer coefficients, developed for uniform flow over an isolated droplet (or particle), in computing the droplet dynamics. This point-particle approach is typically extended to account for higher volume loadings, by incorporating the reaction force, heat, mass transfer from the droplet phase to the fluid phase ('two-way coupling') [2], accounting for the fluid volume displacement by droplet motion by incorporating local volume fraction variations in the governing equations ('volume-filtered' or 'volumetric' coupling) [3], and incorporating models for inter-droplet interactions, droplet breakup [4], and coalescence. Applying this model to realistic spray systems, often results in situations where the main assumption of droplet being subgrid is not satisfied, especially near the injector. Then, the self-disturbance created by the droplet, through two-way and volumetric coupling, can be significant, and alter the flow variables at the droplet location (velocity, mass-fractions, and temperature). However, their undisturbed values are needed for drag, heat and mass transfer models, and are no longer available.

Obtaining the undisturbed flowfield, by correcting for the self-disturbance created by the droplet, has received significant attention recently $[5,6,7,8]$. Majority of these studies are for isothermal, solid particles, and invoke low Reynolds number, Stokes flow solutions. Liu et al. [9] extended this approach for a heated solid particle using similar assumptions. In this work, a general formulation is first derived involving partial differential equations for the self-disturbance field created by a droplet in the momentum and energy equations. Here, droplet evaporation and chemical reactions are neglected; however, extending the idea to include these effects is straightforward. These equations can be solved using the same numerical approach employed 
in the fluid flow solver, and thus can be used in any numerical solver. The model is tested for two simple cases involcing a single isolated droplet (i0 held fixed in a hot, uniform flow, and (ii) falling under gravity in a pool of hot fluid. For low Peclet numbers, it is first shown that the uncorrected velocity and temperature of the droplet predicted by the standard point-particle approach are highly inaccurate, especially for droplet sizes on the order of grid resolution. The fluid flow corrected for self-disturbance is then shown to predict the droplet dynamics accurately. The approach is valid for a range of droplet Reynolds and Peclet numbers, different models for drag force and heat transfer, and can be extended to droplet evaporation and mass transfer. It is also applicable directly to any type of grid (structured or unstructured, isotropic or anistropic grids) and any complex boundary conditions.

\section{Mathematical formulation}

The mathematical formulation is based on mass, momentum, and energy conservation equations in the limit of zero-Mach number. Any acoustic interactions and compressibility effects are neglected and the thermodynamic pressure is assumed constant. In addition, for simplicity of the analysis, temperature-induced density variations within the fluid due inter-phase heat transfer are assumed small; that is, the energy equation is decoupled. Such an assumption is reasonable in absence of chemical reactions, and when buoyancy induced thermal effects are not important. However, note that, the formulation for disturbance field due to presence of droplets as presented here can be easily extended to flows with variable density, multiple species, evaporation, and chemical reactions. The mass, momentum, and energy equations for two-way coupled single droplet system are,

$$
\begin{aligned}
\frac{\partial u_{j}}{\partial x_{j}} & =0 \\
\frac{\partial \rho_{g} u_{i}}{\partial t}+\frac{\partial \rho_{g} u_{i} u_{j}}{\partial x_{j}} & =-\frac{\partial p}{\partial x_{i}}+\frac{\partial}{\partial x_{j}}\left(2 \mu S_{i j}\right)+\dot{S}_{i}, \\
\frac{\partial \rho_{g} h}{\partial t}+\frac{\partial \rho_{g} h u_{j}}{\partial x_{j}} & =\frac{\partial}{\partial x_{j}}\left(\rho_{g} \alpha_{h} \frac{\partial h}{\partial x_{j}}\right)+\dot{S}_{h},
\end{aligned}
$$

where $S_{i j}=\frac{1}{2}\left(\frac{\partial u_{i}}{\partial x_{j}}+\frac{\partial u_{j}}{\partial x_{i}}\right)$ and $\rho_{g}$ is the density of the fluid (assumed constant), $u_{i}$ is the velocity, $\mu$ is the dynamic viscosity, $p$ is the pressure, $h$ is the total enthalpy, $\dot{S}$ are the source terms due to inter-phase coupling. These source terms may be based on advanced models for droplet drag and droplet heating.

The above equations result in velocity, and enthalpy fields that are disturbed by the interaction terms from the droplet phase. The undisturbed flow field (denoted by superscript ()$\left.^{\text {un }}\right)$ can be obtained from the above equations without the inter-phase coupling terms. The difference between the undisturbed and disturbed flow for a single droplet, gives the disturbance flowfield (denoted by superscript ()$^{d}$ ),

$$
u_{i}^{u n}=u_{i}+u_{i}^{d} ; \quad p^{u n}=p+p^{d} ; \quad h^{u n}=h+h^{d} .
$$

Although, the disturbance equations can be derived for a general, multi-species, variable density flow, for simplicity, the equations below are written for the case where density variations within the fluid due inter-phase heat transfer are assumed small; that is, disturbance in density 
is negligible. Then, the disturbance equations can be derived and shown to be,

$$
\begin{aligned}
\frac{\partial u_{j}^{d}}{\partial x_{j}} & =0, \\
\rho_{g} \frac{\partial u_{i}^{d}}{\partial t}+\rho_{g} u_{j} \frac{\partial u_{i}^{d}}{\partial x_{j}} & =-\frac{\partial p^{d}}{\partial x_{i}}+\frac{\partial}{\partial x_{j}}\left(2 \mu S_{i j}^{d}\right)-\dot{S}_{i}, \\
\rho_{g} \frac{\partial h^{d}}{\partial t}+\rho_{g} u_{j} \frac{\partial h^{d}}{\partial x_{j}} & =\frac{\partial}{\partial x_{j}}\left(\rho_{g} \alpha_{h} \frac{\partial h^{d}}{\partial x_{j}}\right)-\dot{S}_{h} .
\end{aligned}
$$

In the above equations, the advective terms give rise to additional terms $\rho_{g} u_{j}^{d} \partial u_{i}^{u n} / \partial x_{j}$ and $\rho_{g} u_{j}^{d} \partial h^{u n} / \partial x_{j}$, which require additional closure between the undisturbed and disturbance fields. However, these terms can be safely neglected by hypothesizing that the spatial variations in the undisturbed flow are negligible compared to those in the disturbed flow, $\partial u_{i}^{u n} / \partial x_{j} \ll \partial u_{i}^{d} / \partial x_{j}$. Note that the nonlinear, advective terms contain the disturbance velocity $\left(u_{i}^{d}\right)$ and enthalpy $\left(h^{d}\right)$ and the two-way, coupled velocity $\left(u_{j}\right)$. The latter is readily available in a two-way coupled simulation from the solution of equations (1-3). The above disturbance equations can be directly solved in addition to the two-way coupled equations (1-3).

However, this requires an additional step involving an expensive pressure Poisson solution for the disturbance pressure, $p^{d}$. To further simplify this solution process, an approximate solution of the disturbance field is proposed that is derived based on low droplet Reynolds number assumption. It is worth noting that, the fluid response to the droplet force, is approximately analogous to the flow that would be generated by the droplet. This is in fact the main assumption employed in the two-way coupled point-particle approach wherein it is assumed that the droplet point-force can approximately produce the same flow as a finite size droplet would do in reality. In the limit of steady state and $R e_{p}<0.1$, a Stokes solution is obtained for the flow created around the actual droplet. In this Stokes regime, the drag on the droplet that experiences slip velocity of $u_{p}^{r e l}$, consists of two terms (i) pressure and (ii) viscous forces as,

$$
F_{\text {drag }}^{\text {Stokes }}=\underbrace{\pi \mu d_{p} u_{p}^{r e l}}_{\text {Pressure force }}+\underbrace{2 \pi \mu d_{p} u_{p}^{r e l}}_{\text {Viscous force }} .
$$

For low particle Reynolds numbers, the expression for these two forces are identical, with viscous force being twice greater than the pressure force. Motivated by this, one can model the contribution of the pressure drag through an effective viscosity and rewrite the Stokes drag force as,

$$
F_{i, \mathrm{drag}}^{\text {Stokes }}=2 \pi \mu_{e f f} d_{p} u_{p}^{r e l} ; \quad \mu_{e f f}=K_{\mu} \mu ; \quad K_{\mu}=1.5 .
$$

Rewriting the Stokes drag in this form facilitates the approximation that the effect of the pressure gradient term in Eq. 6 can be modeled through an equivalent viscous term with an effective viscosity with $K_{\mu}=1.5$ to match the net fluid force in the Stokes limit. It should be noted that the continuity constraint is already embedded in the Stokes solution from which the Stokes drag is obtained. Therefore, it is conjectured that the introduced correction factor will implicitly provide a velocity field that approximately satisfies the continuity equation. Since, the pressure correction term is no longer needed, the continuity equation in Eq.5 is unnecessary, and is only satisfied approximately. The disturbance field due to the droplet forces, can be computed by solving only the momentum equation in each direction with viscous stresses and a modified viscosity through the introduced correction factor of $K_{\mu}=1.5$. This results in a simplified, unsteady advection-diffusion-reaction equation for the disturbance velocity components and enthalpy. These equations can be solved very efficiently to give spatio-temporally accurate solutions. In addition, for higher Reynolds numbers, the correction factor $K_{\mu}$ can also be adjusted to account for different relative contributions of the pressure and viscous forces. 
The approximate disturbance equations then become,

$$
\begin{aligned}
\rho_{g} \frac{\partial u_{i}^{d}}{\partial t}+\rho_{g} u_{j} \frac{\partial u_{i}^{d}}{\partial x_{j}} & =\frac{\partial}{\partial x_{j}}\left(2 K_{\mu} \mu S_{i j}^{d}\right)-\dot{S}_{i}, \\
\rho_{g} \frac{\partial h^{d}}{\partial t}+\rho_{g} u_{j} \frac{\partial h^{d}}{\partial x_{j}} & =\frac{\partial}{\partial x_{j}}\left(\rho_{g} \alpha_{h} \frac{\partial h^{d}}{\partial x_{j}}\right)-\dot{S}_{h} .
\end{aligned}
$$

The above unsteady advection-diffusion-reaction equations are solved in addition to equations (1-3), and the undisturbed velocity and enthalpy fields are evaluated using equations (9). This undisturbed fluid flow variables are then interpolated to the droplet location and used in the drag and heat transfer laws needed to compute the droplet velocity and temperature values.

The above formulation for disturbance field is independent of the model used for the drag force or heat transfer between the fluid and the droplet. In the present work, the following droplet equations are solved;

$$
\frac{d x_{p}}{d t}=u_{p} ; \quad \frac{d u_{i, p}}{d t}=\left(1-\frac{\rho_{g}}{\rho_{p}}\right) g_{i}+\frac{u_{i, @ p}^{u n}-u_{i, p}}{\tau_{p}} ; \quad \frac{d T_{p}}{d t}=\frac{T_{@ p}^{u n}-T_{p}}{\tau_{h}},
$$

where $u_{i, p}$ is the droplet velocity, $T_{p}$ is the droplet temperature, subscript $@ p$ corresponds to the fluid property interpolated to the droplet location, $\tau_{p}$ and $\tau_{h}$ are droplet relaxation and heating time scales. For the present study, the Biot number is assumed small, so temperature variation within the droplet is negligible. The droplet relaxation and heating time-scales, are based on simple Stokes flow solution, although any other advanced model can be used:

$$
\tau_{p}=\frac{\rho_{p} d_{p}^{2}}{18 \mu} ; \quad \tau_{h}=\frac{\rho_{p}}{\rho_{g}} \frac{C_{p, \ell}}{C_{p, g}} \frac{d_{p}^{2}}{12 \alpha_{h}},
$$

where $\rho_{p}$ is the droplet density, $d_{p}$ is the droplet diameter, $C_{p, \ell}$ is the droplet specific heat, and $C_{p, g}$ is the fluid specific heat. The inter-phase source terms are then obtained as,

$$
\dot{S}_{i, @ p}=-m_{p} \frac{u_{i, @ p}^{u n}-u_{i, p}}{\tau_{p}} ; \quad \dot{S}_{h, @ p}=-m_{p} C_{p, \ell} \frac{T_{@ p}^{u n}-T_{p}}{\tau_{h}} .
$$

A consistent Gaussian interpolation kernel is used to interpolate the flow variables from the control volume centers to the droplet locations, and the above source terms at the droplet location to the surrounding control volumes [3].

\section{Results and discussion}

To evaluate the effectiveness of the disturbance correction model, two cases involving a single droplet in a hot fluid are considered: (i) a single droplet is held fixed and at constant temperature in a hot fluid with uniform inflow. The flow variables (velocity and temperature) interpolated to the droplet location are computed for two-way coupled simulation with and without the correction and compared against the undisturbed (one-way) flow, and (ii) a single droplet falling under gravity in a hot, quiescent fluid is computed and the temporal evolution of the droplet speed, temperature are compared for the two-way coupled simulation with and without correction against the one-way coupled simulation. Two different droplet Reynolds numbers are considered for each case.

\section{Fixed Droplet}

In this case, a single droplet is held fixed and at constant temperature in a uniform inflow and temperature higher than the droplet. A domain of of size $[6.4,51.2,6.4]$ with uniform cubic grids of $64 \times 512 \times 64$ is used in the $x, y, z$ coordinates, respectively. A uniform flow with inlet velocity $U_{\text {inlet }}$ is imposed in the vertical $(y)$ direction with a convective outflow boundary condition at 
the other end. Periodic conditions are imposed in the $x$ and $z$ directions. A spherical droplet of size, $d_{p}=0.1 \mathrm{~m}$, is placed at a vertical distance of 7 units from the inlet, and at the center of the domain in $x$ and $z$ directions. The droplet size is selected to be same as the grid resolution. The droplet is held fixed and at a constant temperature of $T_{p}^{*}=\left(T-T_{r e f}\right) / T_{\text {ref }}=0$, where the reference temperature is $T_{r e f}=300 \mathrm{~K}$. The droplet density is set to $\rho_{p}=180 \mathrm{~kg} / \mathrm{m}^{3}$ and the ratio of droplet to fluid specific heat is set to $C_{p, \ell} / C_{p, g}=1.0$. The fluid density, $\rho_{g}=1.0 \mathrm{~kg} / \mathrm{m}^{3}$, dynamic viscosity, $\mu=0.1 \mathrm{~kg} / \mathrm{m} . \mathrm{s}$, and thermal diffusivity is set to $\alpha_{h}=0.1 \mathrm{~m}^{2} / \mathrm{s}$. The initial and inlet fluid temperature are set at $T_{\text {inlet }}^{*}=\left(T-T_{\text {ref }} / T_{\text {ref }}\right)=1$. Only the drag force is considered and gravity is neglected in this case. By varying the inlet velocity $U_{\text {inlet }}=1,10 \mathrm{~m} / \mathrm{s}$, two droplet Reynolds numbers of $R e_{p}=\rho_{g} U_{\text {inlet }} d_{p} / \mu=1,10$ are studied. The corresponding Peclet numbers are $P e_{p}=U_{\text {inlet }} d_{p} / \alpha_{h}=R e_{p} . P r=1,10$.

(a)

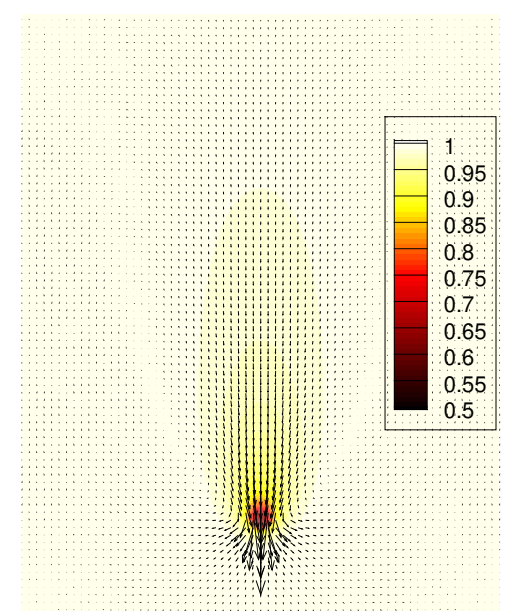

Figure 1. Non-dimensionalized, disturbed fluid temperature contours and vector plot (the inlet velocity is removed from the vertical component): (a) $R e_{p}=1$, (b) $R e_{p}=10$. Only a small region surrounding the droplet is shown.

Figures $1 \mathrm{a}, \mathrm{b}$ show the non-dimensionalized, disturbed fluid temperature contours together with the vector plot for two different Reynolds numbers, respectively. To better visualize the flow field, the inlet velocity is removed from the vertical velocity component while plotting the vector plot. Only a small region surrounding the droplet is shown. It is clearly seen that, with $R e_{p}=10$, the wake of the droplet is longer, but narrower compared to that at $R e_{p}=1$. With one-way coupling, the fluid temperature at the droplet location should be the undisturbed temperature and equal to the inlet temperature. Accordingly, for one-way coupled simulation in this case, the heat transfer from the fluid to the droplet should remain constant. However, with two-way coupling, the fluid temperature surrounding the droplet is affected and lower than the inlet temperature owing to heat transfer from the fluid to the droplet. Thus, the rate of heat transfer from the fluid to the droplet is affected, if this uncorrected fluid temperature is used. It is seen that this effect is much stronger for the lower $R e_{p}$ and $P e_{p}$ case. This is because, for the increased Peclet number (obtained by increasing the inlet fluid velocity), the disturbance created by the droplet is quickly advected away, and its local effect is not as strong. These effects are more pronounced when the droplet size is larger than the grid resolution and diminish in their effect as the droplet size becomes much smaller than the grid resolution (not shown).

Figures $2 a, b$ show the temporal history of the fluid velocity and temperature fields interpolated to the droplet location for the two Reynolds numbers. Plots for before and after the correction are presented. After correction, the non-dimensional velocity and temperature fields at the droplet location are observed to be constant at equal to the undisturbed values, as in a one-way simulation. This shows that the correction model is very effective for both Reynolds numbers. Also shown is the history of the velocity and temperature fields before correction. Their deviation from the corrected values is the error in a two-way coupled simulation. It is seen that, 
(a)

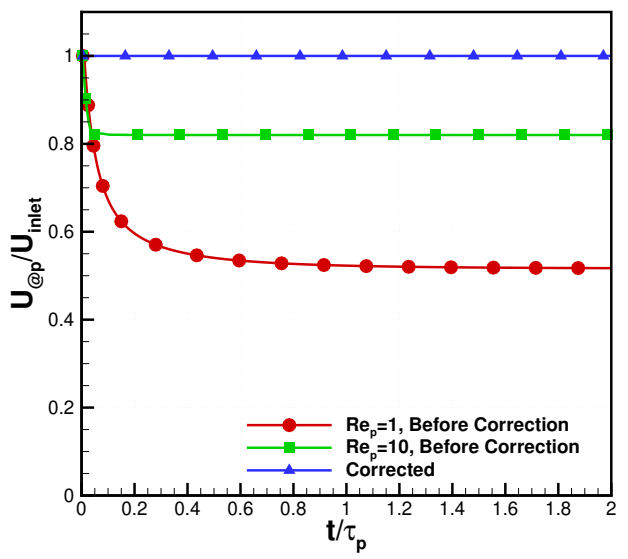

(b)

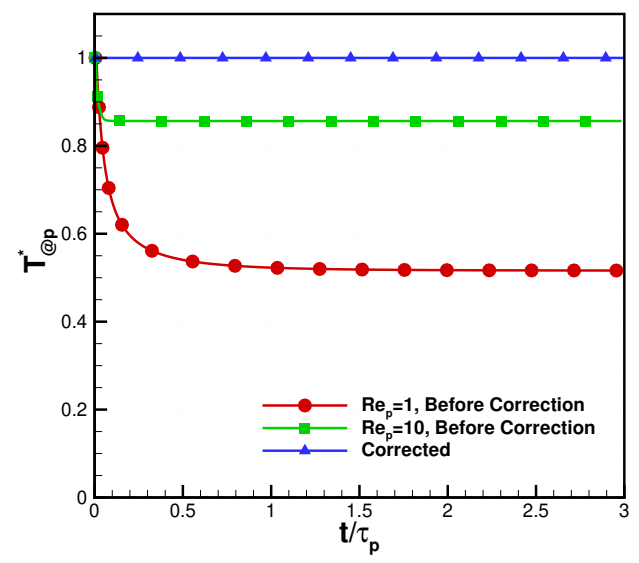

Figure 2. The disturbed flow fields interpolated to the droplet location for two different droplet Reynolds numbers: (a) non-dimensional vertical velocity, (b) non-dimensional temperature $\left(T_{@ p}^{*}=\left(T_{@ p}-T_{r e f}\right) / T_{r e f}\right)$. The corrected and uncorrected fields are shown.

this error is nearly $45 \%$ for $R e_{p}=1$ and decreases to about $15-18 \%$ for $R e_{p}=10$. These are still substantial deviations from the true solution and indicates the necessity of the correction model, especially in the near injector region, where droplets of size on the order of the grid resolution are expected.

\section{Gravitational Settling}

A single droplet settling under gravity in a quiescent, hot fluid is investigated. A triply periodic box of dimensions $6.4 \times 51.2 \times 6.4$ with uniform $64 \times 512 \times 64$ cubic grids are used. A droplet of density $\rho_{p}=180 \mathrm{~kg} / \mathrm{m}^{3}$ and size $d_{p}=0.1 \mathrm{~m}$ is initially placed at $[0,44,0]$ in a fluid of dynamic viscosity $\mu=0.1 \mathrm{~kg} / \mathrm{m} . \mathrm{s}$, thermal diffusivity $\alpha_{h}=0.1 \mathrm{~m}^{2} / \mathrm{s}$, and density of $\rho_{g}=1 \mathrm{~kg} / \mathrm{m}^{3}$. The ratio of droplet to fluid specific heats is set at $C_{p, \ell} / C_{p, g}=1.0$. Gravity is assumed in the vertical direction, and its value is varied to obtain different droplet Reynolds and Peclet numbers. The droplet settling speed $\left(U_{s}\right)$, the particle relaxation time $\left(\tau_{p}\right)$, the droplet Reynolds number $\left(R e_{p}\right)$ and Peclet number $\left(P e_{p}\right)$ are given as,

$$
U_{s}=\left(1-\frac{\rho_{g}}{\rho_{p}}\right) \tau_{p}|g| ; \quad R e_{p}=\frac{\rho_{g} U_{s} d_{p}}{\mu} ; \quad P e_{p}=\frac{U_{s} d_{p}}{\alpha_{h}}=R e_{p} P r
$$

where $g$ is the gravitational acceleration. Two cases with $g=-1.055 \mathrm{~m} / \mathrm{s}^{2}$ and $-10.055 \mathrm{~m} / \mathrm{s}^{2}$ in the vertical $y$ direction are considered resulting in particle Reynolds numbers of 1,10 . Initially, the flow is assumed quiescent and at uniform non-dimensional temperature of 1 , while that of the droplet is assumed as 0 . The non-dimensional temperature is defined as $T^{*}=$ $\left(T-T_{r e f}\right) / T_{r e f}$, where $T_{r e f}=300 \mathrm{~K}$. A small Biot number is assumed, and thus the droplet temperature is assumed uniform.

Figures 3a-d show the temporal history of droplet Reynolds number with corrected and uncorrected fluid flow $(\mathrm{a}, \mathrm{c})$ and non-dimensional fluid temperature at the droplet location with corrected and uncorrected fluid flow $(b, d)$ for two different cases with terminal droplet Reynolds numbers of 1 and 10. It is observed that, when the fluid flow is uncorrected for velocity and temperature disturbance created by the droplet, the settling speeds (and hence the droplet Reynolds numbers) reached by the droplet are much larger than the true settling speeds based on a one-way coupled simulation. This is because, as the droplet modifies the local fluid velocity and temperature, the relative velocity of the droplet is lower than what it would be without any disturbance. Hence, the drag force experienced by the droplet (based on the relative velocity) is lower. As the droplet reaches terminal speed, the drag, buoyancy and weight of the droplet 
must be in balance. With lower drag force, the terminal speed attained by the droplet is larger. From figures 3a,c it can be observed that this overprediction is on the order of $35 \%$ and $10 \%$ for $R e_{p}=1$ and 10, respectively. The error decreases with increased terminal speed because, the droplet moves away form the self-induced disturbance at a faster rate. In both cases, the corrected velocity at the droplet location is obtained to be nearly zero, as it should be in a quiescent, undisturbed flow, and the terminal speeds predicted by the corrected model are nearly identical to the one-way coupled approach. This shows the effectiveness of the correction model. Similar results are obtained for the droplet temperature. As seen in fiqures $3 \mathrm{~b}, \mathrm{~d}$, as the

(a)

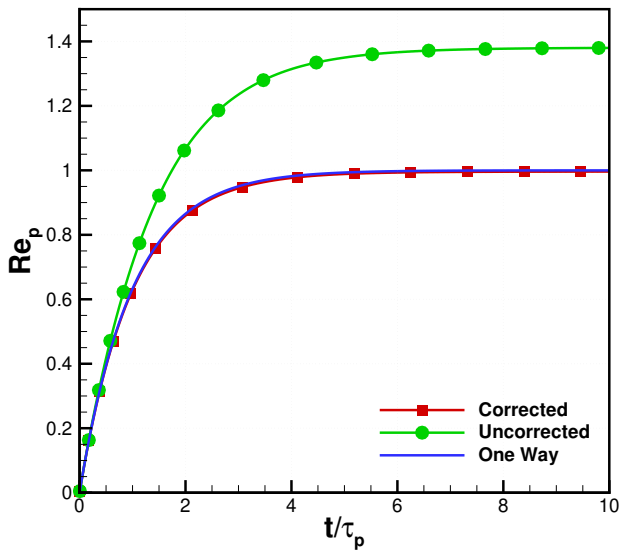

(c)

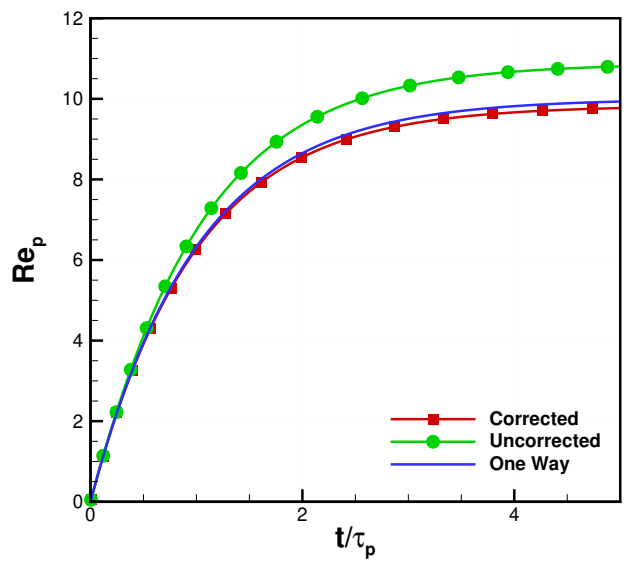

(b)
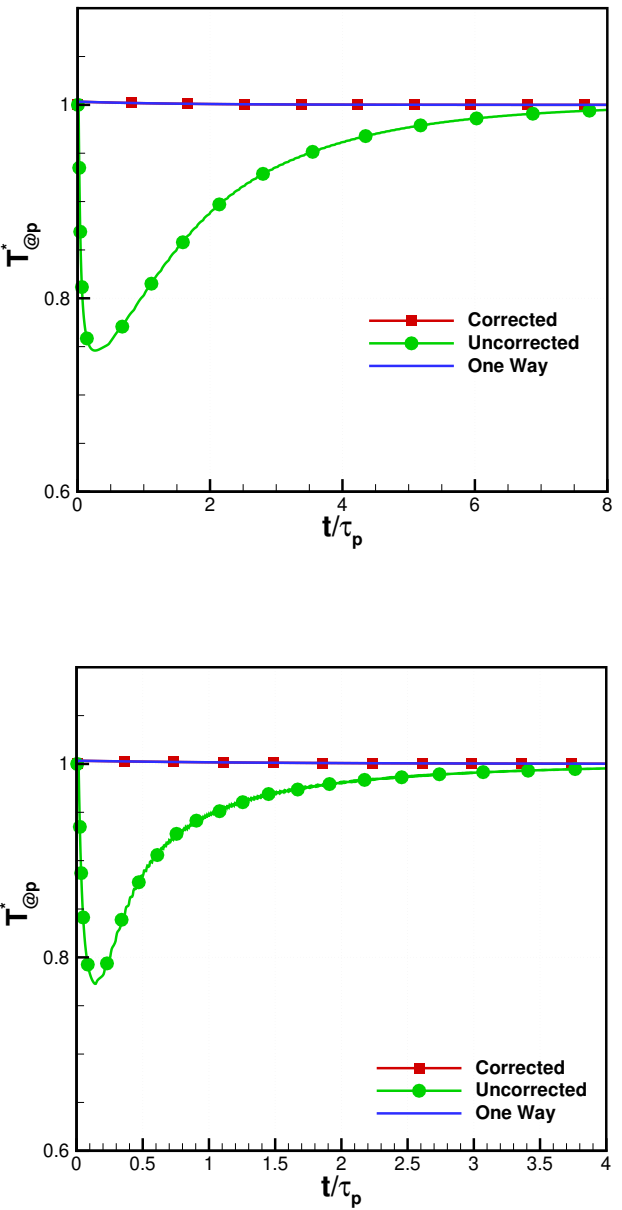

(d)

Figure 3. Time history of droplet Reynolds number $\left(R e_{p}=\rho\left|u_{@_{p}}-u_{p}\right| d_{p} / \mu\right)$ and non-dimensional fluid temperature $\left(T_{@ p}^{*}=\left(T_{@ p}-T_{r e f}\right) / T_{r e f}\right)$ based on the disturbed and corrected fluid velocity and temperature at the droplet location compared with the true, undisturbed solution from one-way coupling: (a,b) $P e_{p}=1$, (c,d) $P e_{p}=10$.

droplet starts from rest, the uncorrected fluid temperature at the droplet location is significantly lower than the one-way coupled value. In this early time, the droplet has not moved significant amount, and yet removes considerable amount of heat from the fluid, which locally results in lower fluid temperature. As the droplet accelerates, and starts to move faster, it is seen that the error in the uncorrected fluid temperature field decreases. In addition, as the droplet starts to heat up, the difference in the fluid temperature and droplet temperature also decrease, and thus lesser amount of heat is removed from the fluid, reducing the amount of disturbance in fluid temperature created by the droplet. In case of droplet falling with higher terminal speed $\left(R e_{p}=10\right)$, the local disturbance created by the droplet is again smaller. With correction, the fluid temperature remains very close to the undisturbed (one-way coupled) value. Since the fluid temperature remains unchanged, the rate of heat transfer to the droplet is higher, compared to the uncorrected case, and the droplet heats up more rapidly (not shown). 


\section{Conclusions}

In a typical point-particle model for droplet sprays, if the size of the droplets is much smaller than the grid resolution, the perturbation created by the droplet through interphase reaction terms in momentum, and energy transport equations is not significant. However, this disturbance becomes significant, when the droplet size becomes comparable to or larger than the grid resolution. In addition, having dense cluster of droplets can also induce significant disturbance. Such a situation is common in several spray systems especially near an injector. Under these conditions, using the disturbed flow field (velocity and temperature) at the droplet location to compute the droplet dynamics can lead to significant errors. An approach that corrects the disturbed flow to obtain the undisturbed flow field was developed and verified.

Governing equations for the disturbance velocity and temperature field were derived. For low Reynolds numbers, unsteady advective-diffusive-reaction equations were obtained for disturbance velocity and temperature fields. These equations are solved in addition to the two-way coupled fluid and particle phase equations to obtain the undisturbed flow field required in solving the droplet equations. The formulation was applied to a simple test cases of (i) a fixed droplet in a uniform heated flow and (ii) a single droplet falling under gravity in a heated, quiescent pool of fluid. For low Reynolds (Peclet) numbers, and droplet size on the order of the grid resolution, the model predicts the undisturbed flowfield accurately. Neglecting to correct for this self-induced disturbance was shown to result in large errors (up to $45 \%$ fluid velocity at the droplet location) at low Reynolds numbers. The error becomes larger with droplet size, but reduces with increase in Reynolds numbers.

The developed model for self-induced disturbance makes use of the baseline algorithm used in the flow solver to solve the governing equations. Thus, it can be easily implemented into any flow solver and is general enough to be applicable to any type of grid (rectilinear, unstructured and uniform or anisotropic grids), interphase interpolation kernels, and bounded or unbounded complex flow configurations. The approach presented here is applicable to a single droplet. For multiple droplets, the disturbance equations can be solved in a small region surrounding each droplet and strategies such as embedded grids or overset grid techniques can be used for these solutions. In addition, the approach can be easily extended to include complex physics of droplet evaporation, and chemical reactions.

\section{Acknowledgements}

SA acknowledges partial support from NSF Award\#1851389 and Honeywell, Inc.

\section{References}

[1] Martin R Maxey and James J Riley. The Physics of Fluids, 26(4):883-889, 1983.

[2] S Elghobashi and GC Truesdell. Physics of Fluids A: Fluid Dynamics, 5(7):1790-1801, 1993.

[3] SV Apte, Krishnan Mahesh, and T Lundgren. International Journal of Multiphase Flow, 34(3):260-271, 2008.

[4] SV Apte, Mikhael Gorokhovski, and Parviz Moin. International Journal of Multiphase Flow, 29(9):1503-1522, 2003.

[5] JAK Horwitz and A Mani. International Journal of Multiphase Flow, 101:74-84, 2018.

[6] S Balachandar, Kai Liu, and Mandar Lakhote. Journal of Computational Physics, 376:160185, 2019.

[7] Fabien Evrard, Fabian Denner, and Berend van Wachem. Journal of Computational Physics: X, p. 100078, 2020.

[8] Pedram Pakseresht, Mahdi Esmaily, and Sourabh V Apte. Journal of Computational Physics, 420:109711, 2020.

[9] Kai Liu, Mandar Lakhote, and S Balachandar. Journal of Computational Physics, 396:596615, 2019. 